$\operatorname{MUTP} / 93 / 24$

\title{
Functional determinants on regions of the plane and sphere
}

\author{
J.S.Dowker \\ Department of Theoretical Physics, \\ The University of Manchester, Manchester, England.
}

\begin{abstract}
The standard formula for the change in the effective action under a conformal transformation is extended to the case when the boundary is piecewise smooth. We then find the functional determinants of the scalar Laplacian on regions of the plane obtained by stereographic projection of the fundamental domains on an orbifolded two-sphere. Examples treated are the sector of a disk and a circular crescent. The effective action on a spherical cap is also determined.
\end{abstract}

October 1993 


\section{Introduction}

Apart from its intrinsic interest, the evaluation of the functional determinant of a differential operator (usually the Laplacian) on a manifold has a number of applications in quantum and string theory as well as in mathematics [1], [2].

Two-dimensional manifolds are the most studied and in this paper we continue our investigation into the orbifolded sphere. The determinant of the scalar Laplacian on a fundamental domain, $\mathcal{M}$ (a spherical triangle), was determined in a previous paper, [3]. Here we wish to employ the conformal transformation technique of projecting the sphere stereographically onto its equatorial plane. The corresponding change in the determinant is given by a standard formula due to Lüscher, Symanzik and Weiss [4], in the first instance, and to Polyakov [5]. We refer to it as the LSWP relation. This will enable the determinants on the planar projections of the fundamental spherical domains to be found.

For the hemisphere this procedure was elaborated and employed by Weisberger [8] to obtain, for example, the determinant on a disk, which would be difficult to find directly in terms of the zeros of Bessel functions, although there is some discussion of Bessel $\zeta$-functions, [6], [7] that might prove useful.

\section{The stereographic conformal transformation}

This is classic material. Let $z=x+i y$ be the coordinates on the equatorial plane of the unit sphere with metric

$$
d s^{2}=4 \frac{d z d \bar{z}}{\left(1+|z|^{2}\right)^{2}}
$$

The metric on the plane is $d z d \bar{z}$. Projecting from the north pole gives the relation with the usual angular coordinates, $z=\cot \theta \exp (i \phi)$.

Imagine the sphere to be tiled according to the quotient $S^{2} / \Gamma$ where $\Gamma$ is one of the extended cyclic, dihedral, tetrahedral, octahedral or icosahedral point subgroups of $\mathrm{O}(3)$. This tiling will project to a partitioning of the plane into a finite number of regions $[\mathbf{9}]$. (Pictures of this can be seen in many places, e.g. [10].) The appearance of the partitioning depends on the choice of projection point and can be altered by rotating the sphere, or by applying a homography to the plane.

\section{The LSWP relation. Application to the orbifolded sphere.}

For classically conformally invariant theories, integrating the conformal anomaly yields the change in the effective action under a conformal, or equivalently, a Weyl, rescaling, $g_{i j} \rightarrow \bar{g}_{i j}=\exp (-2 \omega) g_{i j}$.

Because the domain $\mathcal{M}$ has corners, it is necessary to look again, briefly, at the derivation of the LSWP relation. For simplicity, attention is restricted to Dirichlet conditions. Neumann conditions are treated in the appendix. We emphasise that the transformations considered are conformal everywhere. Schwartz-Christoffel transformations, as used in [11] and [12] for example, are excluded. 
We give our results in terms of the effective action $W$ which is related to the functional determinant by $W=\ln \operatorname{det}\left(-\nabla^{2}\right) / 2=-\zeta^{\prime}(0) / 2$

The standard anomaly equation in two dimensions is

$$
\delta W[\bar{g}]=\frac{1}{4 \pi} C_{1}^{(2)}[\bar{g} ; \delta \omega]
$$

with

$$
C_{1}^{(2)}[g ; f] \equiv \int_{\mathcal{M}} C_{1}^{(2)}(g, x) f(x) g^{1 / 2} d^{2} x
$$

To integrate (2) one needs the explicit form of (3). The derivation of our paper [13] is repeated here. The required expression can be obtained from the simple, integrated heat-kernel coefficient $C_{1}^{(n)}[g ; 1]$ for the conformal operator, $-\nabla^{2}+\xi(n) R$ in $n$-dimensions where $\xi(n)=(n-2) / 4(n-1)$, in the following way.

From the variation of the zeta function, one firstly derives the relation

$$
C_{1}^{(n)}[g ; \delta \omega]=-\left.\frac{1}{n-2} \delta C_{1}^{(n)}[\bar{g} ; 1]\right|_{\omega=0}
$$

which is all one needs, given the precise form of $C_{1}^{(n)}[g ; 1]$.

Calculation shows that, for an $n$-dimensional domain, $\mathcal{M}$,

$$
C_{1}^{(n)}[g ; 1]=\frac{1-6 \xi}{6} \int_{\mathcal{M}} R d V+\frac{1}{3} \sum_{i} \int_{\partial \mathcal{M}_{i}} \kappa d S+\frac{1}{6} \sum_{i<j} \frac{\pi^{2}-\theta_{i j}^{2}}{\theta_{i j}} \int_{I_{i j}} d L
$$

Here $\kappa$ is the extrinsic curvature of the boundary parts and $\theta_{i j}$ is the dihedral angle between the boundary components $\partial \mathcal{M}_{i}$ and $\partial \mathcal{M}_{j}$. For simplicity we have assumed that this angle is constant along the intersection $I_{i j}=\partial \mathcal{M}_{i} \cap \partial \mathcal{M}_{j}$. This is so for a fundamental domain. $d V$ is the invariant volume element on $\mathcal{M}$ and $d S$ and $d L$ are the induced, invariant measures on the boundary and intersection respectively. In two-dimensions the $I_{i j}$ are the vertices of the domain. A vertex has a content of unity.

The difference from the usual expression is the final term so we need only look at this. Under the conformal scaling, $g \rightarrow \bar{g}$, angles are preserved and the only change is in the contents of the $(n-2)$-dimensional intersections, $I_{i j}$. The resulting extra contribution to the variation is thus

$$
\delta C_{1}^{(n)}[\bar{g} ; 1]=-\frac{n-2}{6} \int \frac{\pi^{2}-\theta^{2}}{\theta} \delta \omega d L
$$

where we have symbolically absorbed the summation into the integration. From (4) we get the generalisation of the formula in [13] 


$$
C_{1}^{(n)}[g ; f]=\left(\frac{1}{6}-\xi\right) \int R f d V+\int\left(\frac{1}{3} \kappa f-\frac{1}{2}(n . \partial) f\right) d S+\frac{1}{6} \int \frac{\pi^{2}-\theta^{2}}{\theta} f d L
$$

where $n$ is the inward normal. (Compare also with $[\mathbf{4}],[\mathbf{1 5}]$, and $[\mathbf{1 6}]$, but without the last term.)

The next step is to set the dimension $n$ equal to two, and then to integrate (2). Again we need only discuss the last term in (7) which, for $n=2$, reduces to a sum over the vertices weighted by a factor of $\delta \omega(x)$ evaluated at the vertex.

The integration over $\omega$ can proceed by setting $\delta \omega=\omega \delta t$ and integrating over $t$ from 0 to 1 . The result is

$$
\begin{gathered}
W[\bar{g}, g]=\frac{1}{24 \pi} \int \omega(R+\square \omega) d V+\frac{1}{12 \pi} \int \omega\left(\kappa+\frac{1}{2}(n . \partial) \omega\right) d S- \\
\frac{1}{8 \pi} \int(n . \partial) \omega d S+\frac{1}{24 \pi} \sum_{k} \frac{\pi^{2}-\theta_{k}^{2}}{\theta_{k}} \omega_{k}
\end{gathered}
$$

where the vertices are labelled simply by $k$ and where $\omega_{k}=\omega\left(x_{k}\right)$.

For computational purposes it is sometimes more convenient to rewrite (8) using the conformal relations

$$
\begin{aligned}
& g^{1 / 2} \square \omega=\frac{1}{2}\left(\bar{g}^{1 / 2} \bar{R}-g^{1 / 2} R\right) \\
& h^{1 / 2}(n . \partial) \omega=\bar{h}^{1 / 2} \bar{\kappa}-h^{1 / 2} \kappa .
\end{aligned}
$$

Then

$$
\begin{gathered}
W_{a}[\bar{g}, g]=\frac{1}{48 \pi} \int \omega\left(\bar{g}^{1 / 2} \bar{R}+g^{1 / 2} R\right) d^{n} x+\frac{1}{24 \pi} \int \omega\left(\bar{h}^{1 / 2} \bar{\kappa}+h^{1 / 2} \kappa\right) d^{n-1} x- \\
\frac{1}{8 \pi} \int\left(\bar{h}^{1 / 2} \bar{\kappa}-h^{1 / 2} \kappa\right) d^{n-1} x+\frac{1}{24 \pi} \sum_{k} \frac{\pi^{2}-\theta_{k}^{2}}{\theta_{k}} \omega_{k}
\end{gathered}
$$

where $h$ and $\bar{h}$ are the induced metrics on the boundary.

For $\bar{g}$ we choose the metric of the plane and for $g$ that of the sphere. We assume that the effective action, $W$, on $\mathcal{M}$ is known according to our previous work [3]. We are then able to find the effective action on $\overline{\mathcal{M}}$ using

$$
\bar{W}=W[\bar{g}, g]+W .
$$

By (1) the conformal function is

$$
\omega=\ln \left(\frac{2}{1+|z|^{2}}\right)
$$


Further, $R=2, \bar{R}=0$, and $\bar{g}=\bar{h}=1$ if planar cartesian coordinates, $(x, y)$, are used. Fundamental domains on the orbifolded sphere are geodesic triangles and so $\kappa=0$. Since (great) circles project to circles, $\bar{\kappa}$ is constant over the bounding arcs on the plane.

\section{The hemisphere, cap and disk}

It is instructive to reconsider the hemisphere which was discussed from this point of view by Weisberger [8]. If the sphere is so oriented that $\mathcal{M}$ is the southern hemisphere, and the projection is from the north pole, the projected region, $\overline{\mathcal{M}}$, is the unit, equatorial disk. From (12), $\omega$ vanishes on the equator and the only nonzero contributions in (10) are from the first and third integrals yielding $W[\bar{g}, g]=$ $(\ln 2) / 6-1 / 3$ (see later). Added to the effective action on the hemisphere, this gives the effective action on the unit disk. In order to obtain the result for a disk of radius $a$, we use the fact that, if the linear dimensions of a manifold be scaled by a factor of $\lambda$, the effective action is increased by $-\ln \lambda \zeta(0)$ where $\zeta$ refers to the $\zeta$-function of the original figure. This result can be applied either to the spherical or to the planar domain and we further note that $\zeta(0)$ is preserved by the stereographic projection, equalling $1 / 6$ for the hemisphere. Weisberger's formula is [8]

$$
W_{\mathrm{disk}}=-\zeta_{R}^{\prime}(-1)-\frac{1}{12} \ln 2-\frac{5}{24}-\frac{1}{6} \ln a-\frac{1}{4} \ln \pi .
$$

This expression can be used to determine the effective action on a spherical cap by projecting a disk of radius $a$, centred on the origin say, back onto the unit sphere. Now we have $\kappa=\left(1-a^{2}\right) / 2 a$ and $\bar{\kappa}=1 / a$, whence, from (10),

$$
\begin{gathered}
W[\bar{g}, g]=\frac{1}{6} \frac{a^{2} \ln (2)+\ln \left(1+a^{2}\right)-a^{2}}{1+a^{2}} \\
+\frac{1}{12} \ln \left(\frac{2}{1+a^{2}}\right)\left(1+\frac{4 a \kappa}{\left(1+a^{2}\right)^{2}}\right)-\frac{1}{4}\left(1-\frac{4 a \kappa}{\left(1+a^{2}\right)^{2}}\right) .
\end{gathered}
$$

Combined with the disk expression (13) this gives the Dirichlet effective action on a cap of area $2 \pi \sigma=4 \pi a^{2} /\left(1+a^{2}\right)$ of a unit sphere as

$W_{\text {cap }}^{\mathrm{D}}(\sigma)=-\zeta_{R}^{\prime}(-1)-\frac{5}{24}-\frac{1}{4} \ln 2 \pi+\frac{1}{12}((3 \sigma-1)(3-\sigma)+\sigma(2-\sigma) \ln (2-\sigma)-\ln \sigma)$.

The corresponding Neumann result turns out to be

$$
W_{\mathrm{cap}}^{\mathrm{N}}(\sigma)=-\zeta_{R}^{\prime}(-1)+\frac{7}{24}+\frac{1}{4} \ln 2 \pi+\frac{1}{12}\left(3 \sigma^{2}-8 \sigma+3+\sigma(2-\sigma) \ln (2-\sigma)+5 \ln \sigma\right) .
$$

Of course it is not necessary to go through the intermediary of mapping to the plane. One can project directly to a concentric sphere. However, we always prefer to use unit spheres. 
It should be noted that $W_{\text {cap }}(\sigma)$ diverges as the cap becomes very small but not as it tends to the complete sphere. In this limit $\sigma=2$ with,

$$
W_{\text {cap }}^{\mathrm{D}}(2)=-\zeta_{R}^{\prime}(-1)+\frac{5}{24}-\frac{1}{4} \ln 2 \pi-\frac{1}{12} \ln 2
$$

and

$$
W_{\text {cap }}^{\mathrm{N}}(2)=-\zeta_{R}^{\prime}(-1)+\frac{5}{24}+\frac{1}{4} \ln 2 \pi+\frac{5}{12} \ln 2 .
$$

Figures 1 and 2 show some relevant plots. The Dirichlet values exhibit minima at $\sigma \approx 0.09525$ and $\sigma \approx 1.87667$.

It is interesting to consider another way of introducing nonunit disks. If the normal to the hemisphere's flat surface makes an angle of $\alpha$ with the polar axis, the projection is a disk of radius $\sec \alpha$ whose centre is offset from the origin by a distance of $\tan \alpha$. We leave it as an exercise to perform the necessary integrations in (10) and to obtain agreement with (13).

Instead of rotations of the sphere, we can use homographies of the plane, $z \rightarrow$ $f(z)=(a z+b) /(c z+d)$. The LSWP relation in this case is fairly standard. Both $R$ and $\bar{R}$ are zero and the conformal function is

$$
\omega=-\ln \left|f^{\prime}(z)\right|
$$

For a rotation through $\alpha$ about a horizontal axis, $f$ is

$$
f(z)=\frac{\cos (\alpha / 2) z-\sin (\alpha / 2)}{\sin (\alpha / 2) z+\cos (\alpha / 2)}
$$

although it is not essential to use this specific form.

Substitution of the relevant geometry into (8) produces the integral

$$
\frac{1}{24 \pi} \int_{0}^{2 \pi} \ln (1+\sin \alpha \cos \theta)\left(\frac{\cos \alpha}{1+\sin \alpha \cos \theta}+1\right) d \theta=\frac{1}{6} \ln \cos \alpha
$$

This is an over-elaborate way of evaluating $\zeta(0)$ on the disk.

\section{Some other fundamental domains}

For a generalisation of the hemisphere, we turn to the dihedral group, the fundamental domain of which is a digon, or lune, of angle $\alpha=\pi / q$. If the rotation axis is set along the East-West direction, the projection from the north pole is a circular crescent and the tiling of the southern hemisphere produces an onion-like decomposition of the equatorial disc. As a typical example we take the crescent with sides formed by half the equator and an arc of the circle of radius $\sec \alpha$ and centre $(\tan \alpha, 0)$. From (10) we find, after inserting the values of $\omega, \bar{\kappa}=\cos \alpha$,

$$
W[\bar{g}, g]=\frac{1}{24 \pi} \int \omega d V+\frac{1}{24 \pi} \int \omega \bar{\kappa} d S-\frac{1}{8 \pi} \int \bar{\kappa} d S
$$




$$
\begin{aligned}
= & \frac{1}{24 \pi} \int_{\pi / 2}^{3 \pi / 2} \int_{r(\theta)}^{1} \ln \left(\frac{2}{1+r^{2}}\right) \frac{4 r d r d \theta}{\left(1+r^{2}\right)^{2}}- \\
& \frac{\cos \alpha}{24 \pi} \int_{\pi / 2}^{3 \pi / 2} \ln \left(\frac{2}{1+r(\theta)^{2}}\right)\left(r^{2}(\theta)+r^{\prime 2}(\theta)\right)^{1 / 2} d \theta-\frac{\alpha}{4 \pi},
\end{aligned}
$$

where $r(\theta)$ is the radius of the inner arc. There are no contributions from the corners because $\omega$ vanishes there.

Circle geometry gives

$$
r^{2}(\theta)-2 r(\theta) \tan \alpha \cos \theta-1=0
$$

so

$$
r(\theta)=\tan \alpha \cos \theta+\left(1+\tan ^{2} \alpha \cos ^{2} \theta\right)^{1 / 2} .
$$

Performing the $r$-integration and combining the two $\theta$-integrands we find, writing $r$ for $r(\theta)$ and after a little algebra,

$$
W[\bar{g}, g]=\frac{1}{12 \pi} \int_{\pi / 2}^{3 \pi / 2} \frac{1-r^{2}}{1+r^{2}} \ln \left(\frac{2}{1+r^{2}}\right) d \theta-\frac{\alpha}{3 \pi} .
$$

It does not appear that this can be taken further analytically.

The relation

$$
\frac{r^{2}-1}{r^{2}+1}=\frac{\tan \alpha \cos \theta}{\left(1+\tan ^{2} \alpha \cos ^{2} \theta\right)^{1 / 2}}
$$

is useful and we note that the area of the crescent is

$$
A_{\text {crescent }}=2 \alpha+2 \tan \alpha-(\pi-2 \alpha) \tan ^{2} \alpha .
$$

The effective action on a spherical lune was determined in $[\mathbf{3}]$ and can be combined with (24) according to (11) to give the action on the crescent. Some Dirichlet values are displayed in Figure 3.

The extended dihedral group is another, more easily treated case. The fundamental domain this time is a spherical triangle with angles $\pi / 2, \pi / 2$ and $\pi / q$. The value $q=1$ corresponds to a quartersphere and coincides with the $q=2$ dihedral case discussed above.

With the cyclic rotation axis as the polar axis, a fundamental domain having the south pole as the vertex of angle $\pi / q$ projects to a sector of angle $\pi / q$ of the unit equatorial disk. $\bar{\kappa}$ is zero on the bounding radii of the sector and unity on the circular arc.

Looking at (10) for $W[\bar{g}, g]$ we see that it reduces to

$$
W[\bar{g}, g]=\frac{1}{24 \pi} \int \omega d V-\frac{1}{8 q}+\frac{q^{2}-1}{24 q} \ln 2
$$




$$
\begin{gathered}
=\frac{1}{6 \pi} \int_{0}^{\pi / q} \int_{0}^{1} \ln \left(\frac{2}{1+r^{2}}\right) \frac{r d r d \theta}{\left(1+r^{2}\right)^{2}}-\frac{1}{8 q}+\frac{q^{2}-1}{24 q} \ln 2 \\
=\frac{1}{12 q}\left(\ln 2-\frac{1}{2}\right)-\frac{1}{8 q}+\frac{q^{2}-1}{24 q} \ln 2 \\
=-\frac{1}{6 q}+\frac{q^{2}+1}{24 q} \ln 2 .
\end{gathered}
$$

Combining this at $q=1$ with the value obtained in [3] for the quartersphere, we find the Dirichlet effective action on half a unit disk to be,

$$
W_{\mathrm{D}}=\frac{1}{24}-\frac{1}{3} \ln 2-\frac{1}{2} \zeta_{R}^{\prime}(-1)-\frac{1}{4} \ln \pi
$$

Figure 4 plots the values for other values of $q$.

There are many other possibilities for projection and it does not seem worthwhile giving an exhaustive treatment. We leave the other orbifolds for possible consideration at another time.

\section{Appendix}

A zero-mode occurs for Neumann conditions and there is the further complication that conformal invariance is lost in $n$-dimensions. To overcome this, we consider, as in [17], Robin conditions

$$
\left.(\psi+(n . \partial)) \phi\right|_{\partial \mathcal{M}}=0
$$

where $\psi$ is a function, somewhat similar to a gauge potential, defined on the boundary, whose conformal behaviour is designed to compensate for terms introduced by the derivative. This behaviour is, under $g \rightarrow \bar{g}$,

$$
\psi \rightarrow \bar{\psi}=e^{\omega}\left(\psi+\frac{1}{2}(n-2)(n . \partial) \omega\right)
$$

We can determine the folded coefficient $C_{1}^{(2)}[g ; f]$ in the Neumann case following the procedure outlined in section 3. The simple integrated Robin coefficient in $n$ dimensions is

$$
C_{1}^{(n)}[g ; 1]=\frac{1-6 \xi}{6} \int_{\mathcal{M}} R d V+\sum_{i} \int_{\partial \mathcal{M}_{i}}\left(\frac{1}{3} \kappa-2 \psi\right) d S+\frac{1}{6} \sum_{i<j} \frac{\pi^{2}-\theta_{i j}^{2}}{\theta_{i j}} \int_{I_{i j}} d L
$$

Actually this has been derived only for smooth boundaries but since any extra terms are due to the local influence of the intersections, $I_{i j}$, dimensional arguments show that there can be no mixing between $\psi$ and these terms. 
Because conformal invariance is being maintained, equation (4) is still valid and, from (30), we see that there will be a cancelling factor of $(n-2)$ to give the required coefficient,

$$
C_{1}^{(n)}[g ; f]=\frac{1-6 \xi}{6} \int R f d V+\int\left(\frac{1}{3} \kappa-2 \psi+\frac{1}{2}(n . \partial)\right) f d S+\frac{1}{6} \int \frac{\pi^{2}-\theta^{2}}{\theta} f d L .
$$

In two dimensions, Neumann conditions are conformally invariant. We can then consistently set $\psi$ to zero and integrate the anomaly equation

$$
\delta W[\bar{g}]=\frac{1}{4 \pi} C_{1}^{(2)}[\bar{g} ; \delta \omega]+\frac{1}{2} \delta \bar{V}
$$

using (32) to give

$$
\begin{gathered}
W[\bar{g}, g]=\frac{1}{2} \ln \left(\frac{\bar{V}}{V}\right)+\frac{1}{24 \pi} \int \omega(R+\square \omega) d V+ \\
\frac{1}{12 \pi} \int \omega\left(\kappa+\frac{1}{2}(n . \partial) \omega\right) d S+\frac{1}{8 \pi} \int(n . \partial) \omega d S+\frac{1}{24 \pi} \sum_{k} \frac{\pi^{2}-\theta_{k}^{2}}{\theta_{k}} \omega_{k} .
\end{gathered}
$$

\section{References}

[1] B.Osgood, R.Phillips and P.Sarnak, J. Funct. Anal. 80 (1988) 148.

[2] T.P.Branson and B.Ørsted Proc. Am. Math. Soc. 113 (1991) 669.

[3] J.S.Dowker Effective action in spherical domains. Comm.Math.Phys. to be published.

[4] M.Lüscher, K.Symanzik and P.Weiss Nucl. Phys. B173 (1980) 365.

[5] A.M.Polyakov Phys. Lett. 103B (1981) 207.

[6] E.C.Obe J. of Math. Analysis and Appl. 52 (1975) 648.

[7] K.B.Stolarsky Mathematika 32 (1985) 96.

[8] W.I.Weisberger Comm. Math. Phys. 112 (1987) 633.

[9] F.Klein Vorlesungen über das Ikosaeder Teubner, Leipzig (1884).

[10] A.R.Forsyth Functions of a complex variable Cambridge University Press, Cambridge (1893).

[11] J.S.Dowker and K.Worden Class. Quant. Grav. 9 (1992) 289.

[12] E.Aurell and P.Salomonson On functional determinants of Laplacians in polygons and simplices, Stockholm (April 1993).

[13] J.S.Dowker and J.P.Schofield J. Math. Phys. 31 (1989) 808.

[14] L.Bukhbinder, V.P.Gusynin and P.I.Fomin Sov. J. Nucl. Phys. 44 (1986) 534.

[15] O.Alvarez Nucl. Phys. B216 (1983) 125.

[16] T.P.Branson and P.B.Gilkey Comm. Partial Diff. Equations 15 (1990) 245.

[17] G.Kennedy, R.Critchley and J.S.Dowker Ann. Phys. 125 (1980) 346. 


\section{Figure Captions}

Figures 1 and 2. Effective action, W, for Dirichlet and Neumann conditions on a spherical cap of area $2 \pi \sigma$.

Figure 3. Effective action for Dirichlet conditions on a planar crescent of angle $\pi / q$ and side curvatures of 1 and $\cos (\pi / q)$

Figure 4. Dirichlet effective action on a sector of angle $\pi / q$ of a unit disk. 